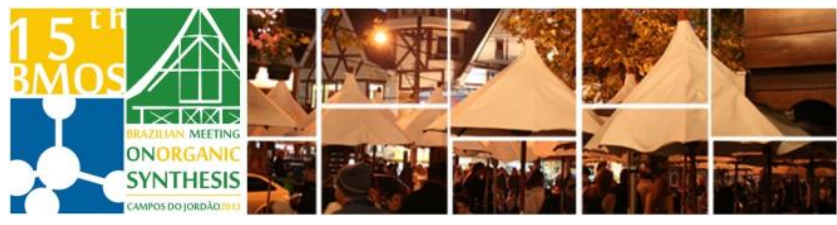

\title{
On the Ugi mechanism probed by ESI-MS(/MS)
}

\section{Gisele A. Medeiros, ${ }^{\star a}$ Wender A. da Silva, ${ }^{a}$ Giovana A. Bataglion, ${ }^{b}$ Davi A. C.} Ferreira, ${ }^{a}$ Heibbe C. B. de Oliveira, ${ }^{a}$ Marcos N. Eberlin, ${ }^{b}$ and Brenno A. D. Neto, ${ }^{a}$

a Laboratory of Medicinal and Technological Chemistry, IQ-UnB, Brasilia, Brazil.

${ }^{b}$ University of Campinas - UNICAMP, Campinas, SP, Brazil. *giselea.medeiros@gmail.com

Keywords: Ugi MCR, Charge tags, ESI-MS(/MS).

\section{INTRODUCTION}

The interest over multicomponent reactions (MCR) has increased substantially. ${ }^{1}$ Among MCR of four components, the Ugi Reaction (U4CR) has attracted special interest owing to its versatility and facility to generate new libraries of bioactive compounds. ${ }^{2}$

\section{RESULTS AND DISCUSSION}

Scheme 1 shows the two charge-tagged reagents used to probe the mechanism and the two accepted reaction pathways for the U4CR.

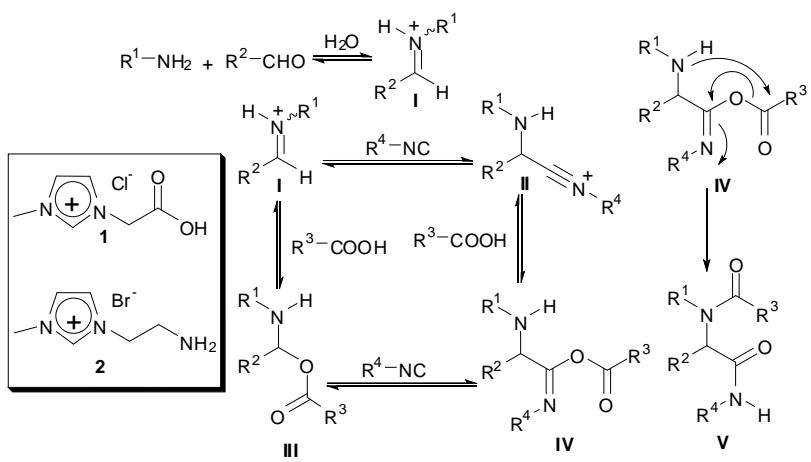

Scheme 1. Charge-tagged reagents and the two accepted reaction patways for the Ugi four-component reaction.

Figures 1 and 2 show the ESI(+)-MS/MS (from Water Synapt) spectra of the ions of $m / z 343$ and of $\mathrm{m} / \mathrm{z} 221$, respectively.

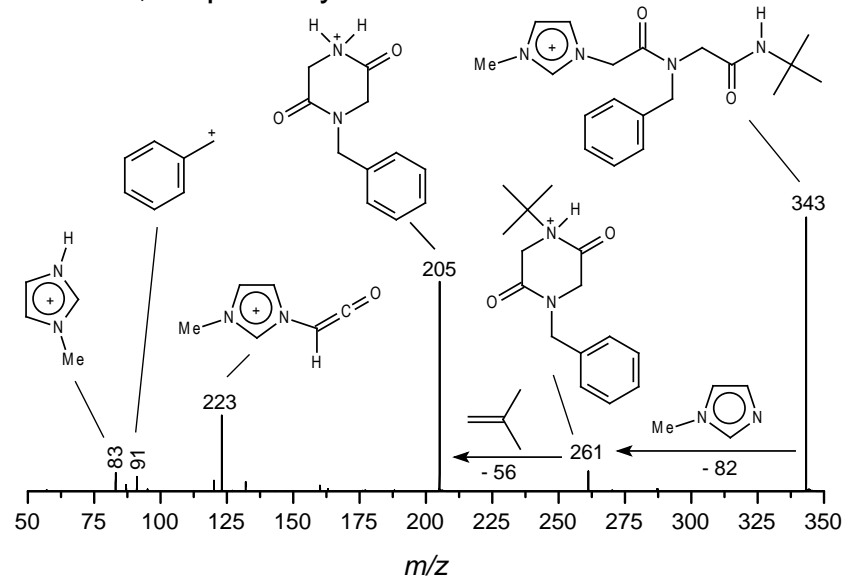

Figure 1. $\mathrm{ESI}(+)-\mathrm{MS} / \mathrm{MS}$ of the ion of $\mathrm{m} / \mathrm{z} 343$ (intermediate V).

It is noted that these two ions could only be detected due to the presence of the charge tags.
The final rearrangement was also evaluated by means of DFT calculations (Figure 3).

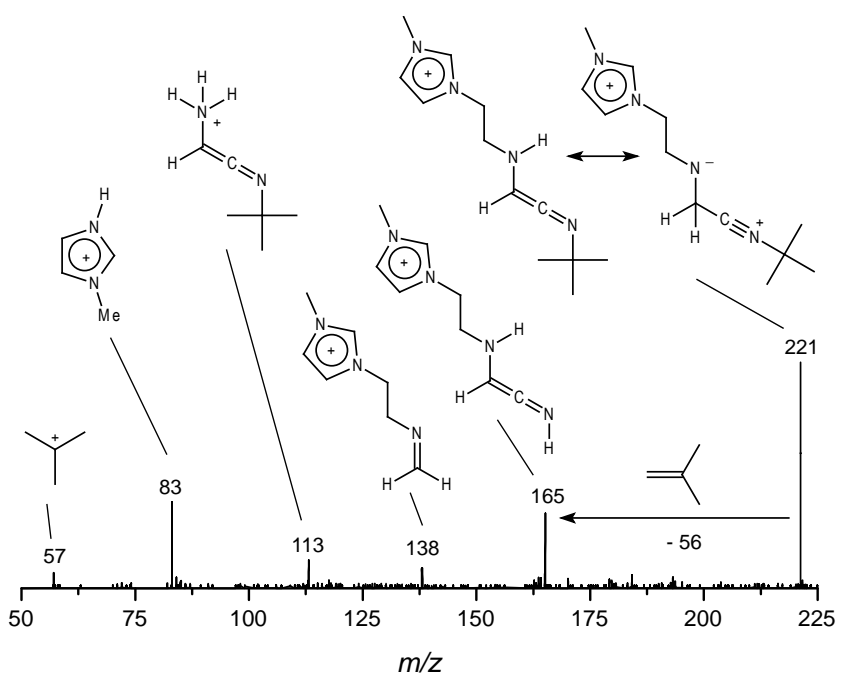

Figure 2. $\mathrm{ESI}(+)-\mathrm{MS} / \mathrm{MS}$ of the ion of $\mathrm{m} / z 221$ (intermediate II).

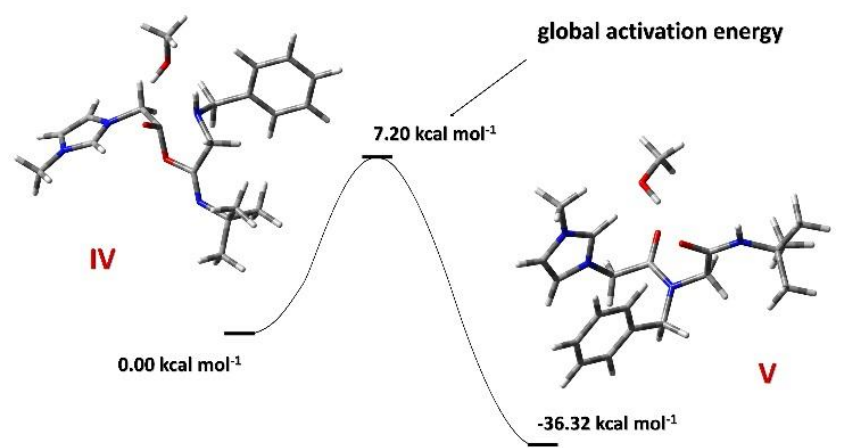

Figure 3. Rearrangement from IV to V. M062X/6-311+G(2d, $2 p) / / M 062 X / 6-31+G(d, p)$ level of theory. Methanol has been considered with explicit treatment for the calculated structures.

\section{CONCLUSION}

The iminium mechanism seems to be the most probable once its intermediates are the most stable.

\section{ACKNOWLEDGEMENTS}

FAPDF, CNPq, CAPES, FAPESP, DPP-UnB.

\section{REFERENCES}

1 Biggs-Houck J. E.; Younai, A.; Shaw J. T. Curr. Opin. Chem. Biol. 2010 14,371 .

${ }^{2}$ C. de Graaff; E. Ruijter; R. V. A. Orru Chem. Soc. Rev. 2012, 41, 3969. 